\title{
Concierto de Chira, para tiorba y orquesta de guitarras. Una propuesta musical para sanar el olvido crónico
}

\author{
Concierto de Chira for Theorbo and Guitar Orchestra. A \\ Musical Proposal to Heal Chronic Oblivion
}

Mario Solera Salas

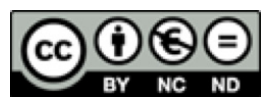

Esta obra está bajo una licencia Creative Commons Reconocimiento-No comercial-Sin Obra Derivada 
Artículo

\title{
Concierto de Chira, para tiorba y orquesta de guitarras. Una propuesta musical para sanar el olvido crónico
}

\author{
Concierto de Chira for Theorbo and Guitar Orchestra. A \\ Musical Proposal to Heal Chronic Oblivion
}

\author{
Mario Solera Salas ${ }^{1}$ \\ Universidad de Costa Rica \\ Costa Rica
}

Recibido: 23 de agosto del 2017 Aprobado: 21 de setiembre del 2017

\begin{abstract}
Resumen
A partir de las particularidades compositivas del Concierto de Chira, para tiorba y orquesta de guitarras, el texto pretende aclarar algunos espacios de la historia costarricense, como la construcción de nación desde la óptica del poder hegemónico ubicado en el Valle Central y la conformación de una sociedad, mayoritariamente machista, las cuales han producido la invisibilización, del actuar de las mujeres en el concierto costarricense, de una región en particular y de una música regional. La tiorba, instrumento de origen aristocrático, extraño en estas tierras, dialoga con las guitarras, instrumento popular por antonomasia, es este espacio dialógico, el que permite develar, desde distintas perspectivas, parte de la historicidad del pueblo costarricense.
\end{abstract}

Palabras clave: música; tiorba; guitarra; concierto; tamborito chiricano

1 Director de la Sede Regional del Pácifico "Arnoldo Ferreto Segura" (Universidad de Costa Rica). Máster en Administración Educativa por la Universidad de Costa Rica. Correo electrónico: mario.solera@ucr.ac.cr 


\begin{abstract}
From the musical characteristics of the Concierto de Chira, for theorbo and guitar orchestra, the text seeks to clarify some areas of Costa Rican history, such as the construction of a nation from the perspective of the hegemonic power located in the Central Valley and the conformation of a Society, mostly male chauvinist, which have produced the invisibilization of the women act in the Costa Rican concert, of a particular region and of a regional music. The theorbo, an instrument of aristocratic origin, strange in these lands, dialogues with guitars, a popular instrument for antonomasia and it is this dialogical space, which allows us to unveil, from different perspectives, part of the historicity of the Costa Rican people.
\end{abstract}

Keywords: music; theorbo; guitar; concert; chirican tamborito 


\section{Introducción}

Costa Rica es un pequeño país del istmo centroamericano. San José, su capital, se ubica en el Valle Central, una depresión geográfica limitada por dos de los sistemas montañosos más importantes del país. En sus más de 3000 km² alberga, además de la capital, tres cabeceras de provincia. Desde sus 1200 metros de altitud media, el poder hegemónico ha decidido como debe ser la nación costarricense. En este accionar, las personas que habitan provincias y ciudades ubicadas en la periferia del Valle Central han sido sistemáticamente invisibilizadas, silenciadas y olvidadas. Por otra parte, la nación costarricense no escapa al estigma de la falocracia, de esta manera, ha silenciado, en muchas oportunidades, la voz académica, cultural y solidaria de insignes mujeres y prácticas culturales que, por tradición, son lideradas por mujeres.

El Concierto de Chira para tiorba y orquesta de guitarras se estrenó el lunes 18 de agosto de 2014, en el marco del III Festival Internacional de Música con Plectro, celebrado en la Sede del Pacífico de la Universidad de Costa Rica, en el que yo participe en la interpretación de la tiorba y fui acompañado por la Orquesta de guitarras "Fascinatio citharis", de Brühl, Alemania, bajo la dirección de Walter Barbarino. La obra fue escrita a propósito para estos instrumentos. La tiorba es un instrumento que fue silenciado por los cambios de estilo sucedidos en la Europa del siglo XVIII y la guitarra fue olvidada por varios siglos.

El Concierto de Chira pretende crear un espacio para evidenciar y reflexionar sobre el impacto del poder hegemónico ejercido por el Valle Central, a largo de la corta historia de la nación costarricense y la actitud silenciadora, invisibilizadora de la falocracia costarricense. La obra, desde la perspectiva de Jodorowsky y Costa (2011) se propone llamar la atención sobre la amnesia crónica de la sociedad costarricense que, para el caso particular, olvidó el tamborito chiricano puntarenense y otras manifestaciones culturales vernáculas; ha olvidado a mujeres brillantes en su actuar y en su intelectualidad; ha olvidado la isla de Chira y sus habitantes, así como también da la espalda, sistemáticamente, a las culturas originales de este territorio. Se adhiere el concierto al pensamiento de Jodorowsky cuando a la pregunta ¿cuál es la finalidad del arte? Responde: "si el arte no sana, no es arte" (Jodorowsky, 2009, p. 218)

El Valle Central, de manera autoritaria y de acuerdo con sus propias filiaciones en materia de cultura, definió lo que es "ser costarricense", los símbolos que identifican a la Nación y otorgan cohesión. Curiosamente, la mayoría de estos responden al entorno natural del Valle Central de corte europeizante, como en el caso de las Bellas Artes, Fumero (2005) apunta:

A inicios del siglo XX la identidad costarricense se reconstruyó a la luz de los conceptos del romanticismo y liberalismo decimonónico lo que supuso una nueva cultura cuyos patrones se trazaron en producciones consideradas nacionales. El movimiento se apoyó en

ESCENA. Revista de las artes, 2018, Vol. 78, Núm. 1 (julio-diciembre), pp. 161-178. ISSN 2215-4906 
el proyecto liberal asociado a la noción de progreso: urbano, europeizado y laico, visión que definió la cultura concebida como bellas artes (Fumero, 2005, p. 6).

\section{La isla de Chira}

Continuando con las ideas esbozdas en la introducción, se debe decir que la isla de Chira ha sido víctima de la invisibilización del Valle Central, a pesar de la riqueza histórica y natural que ofrece. Este lugar, bien conocido por los pueblos originarios de la península de Nicoya y lugares aledaños, aparece en la historia de la nación costarricense cuando el célebre y temido Gobernador de Castilla, Pedro Arias de Ávila y Ortiz de Valdivieso, conocido como Pedrarias Dávila, se entera que su lugarteniente, Francisco Hernández de Córdoba -fundador de Villa Bruselas, la primera comarca española en Costa Rica- se había aliado a Hernán Cortés y desde Nicaragua comenzaba a levantarse en su contra. Por esto, Pedrarias Dávila decide viajar al vecino país del norte, lugar donde se atrincheraba su rebelde lugarteniente. De esta manera, el 16 de marzo de 1526, gracias a que las condiciones naturales de la Isla de Chira ofrecían un sitio de descanso y proveían de alimentos, llegan a ella el Gobernador de Castilla de Oro -hoy día Panamá-, el señor Pedrarias Dávila y su ejército (Ibarra, 2001), para descansar, recobrar fuerzas y desde ahí recuperar Villa Bruselas y proseguir a Nicaragua para capturar al rebelde (Vitoria, 2004).

Así se registra, por primera vez en la historia costarricense la Isla de Chira, no obstante, y como se dijo anteriormente, el lugar era bien conocido por los pueblos originarios de la región. Fue habitada por los indios chorotegas quienes llegaron al istmo entre los siglos VII y VIII de nuestra era (Isla de Chira, s.f.A). Provenían de México, específicamente de Cholula, de ahí su nombre cuyo significado, según Kaufman, es originario de Cholula (Kaufman, 1991). La palabra "chira" en Costa Rica designa a la espata que envuelve la inflorescencia del plátano, cuyo color es púrpura (Ferrero, 2002). También, refiere a una leyenda chorotega en la que se narra que la isla fue un regalo de bodas para Chira, hija de un cacique indígena y primera habitante de la isla (Isla de Chira, s.f.B).

La isla, con sus 43 kilómetros cuadrados, es la más grande del Golfo de Nicoya. De acuerdo con el censo 2011, la región cuenta con 1576 habitantes (INEC, 2012), quienes se dedican a actividades de subsistencia y comerciales, como extracción de sal, pesca artesanal y una incipiente actividad turística. Hasta la década de los ochenta del siglo pasado, se llevó la electricidad vía conexión submarina desde tierra firme. El acueducto que la conectó con el abastecimiento nacional de agua potable fue construido en el año 2000. Antes de este, las y los habitantes debían construir pequeños pozos artesanales para el abastecimiento de agua (Isla de Chira, s.f.C). Asimismo, la primera escuela, la Escuela de Chira, se fundó en 1926, posteriormente, se fundaron la Escuela Bocana en el año 1958 y, años más tarde, la Escuela Montero y Palito. El Liceo se fundó en 1996, la institución vino a solventar el 
problema de aquellas y aquellos estudiantes que, si querían continuar con la educación secundaria debían hacerlo fuera de la isla, esto con los inconvenientes de los escasos medios de transporte, la pérdida de lecciones y la exposición a riesgos de naufragio a causa de las inclemencias del tiempo (Isla de Chira, s.f.C).

Las personas de la isla, si bien es cierto, desarrollan su vida ahí, no pueden ser dueños de las tierras que habitan, ya que estas se les dan por medio de concesiones municipales. En 1998, se establece el primer puesto de salud "Equipo Básico de Atención Integral en Salud" (EBAIS) y, en noviembre de ese mismo año, la isla se constituye en el distrito decimotercero del Cantón Central de la provincia de Puntarenas, lo que permite su ingreso en la lista de beneficiados del programa Triángulo de Solidaridad, situación que le permite impulsar algunos proyectos de desarrollo comunal (Isla de Chira, s.f.B).

Si bien es cierto, la historia de esta isla es larga y rica en acontecimientos, se observa que obtuvo atención estatal hasta hace muy pocos años, tanto en la cobertura de servicios básicos como en la creación de instituciones educativas, de salud y en los medios de acceso desde tierra firme. Según narran habitantes de la isla, hasta hace poco, los únicos medios de transporte interno eran el caballo y la carreta. Para viajar a Puntarenas usaban embarcaciones a vela, durando alrededor de cinco horas en el trayecto. Para los quehaceres domésticos debían sacar el agua de los pozos ubicados, generalmente, a un kilómetro de distancia de sus hogares. Cocinaban con leña y quemaban el excremento seco del ganado para ahuyentar a los mosquitos (Isla de Chira, s.f.E)

Para el colectivo costarricense, la Isla de Chira no es un destino turístico, mucho menos un destino habitacional o de trabajo, únicamente es un poco de tierra que sobresale de las aguas al final del Golfo de Nicoya que se le recuerda solo como la isla más grande de este golfo. En realidad, Chira en el imaginario costarricense es tan solo una isla habitada por un poco de coterráneas y coterráneos, desconocida y olvidada.

\section{Tiorba, guitarra y tamborito}

De igual manera que la isla, la tiorba y la guitarra, en diferentes momentos, fueron olvidadas. La tiorba es un instrumento de 14 cuerdas, con una cuerda pulsada. Es parte de la familia de los archilaúdes y como este, junto al laúd renacentista y barroco, cayó en el olvido después del siglo XVIII. Se diferencia de los archilaúdes por contar con un brazo de mayor longitud (tiro) y por tener un segundo clavijero, al cual se sujetan cuerdas de mayor extensión con la finalidad de producir notas de registro grave. Otra diferencia, es el uso de cuerda sencilla en la tiorba, a diferencia de los laúdes y el chitarrone que usaban cuerdas dobles, llamadas "orden". 
De acuerdo con el filósofo y teórico musical francés Marin Mersenne, la tiorba fue inventada por Antonio Bardi, I/ Bardella, alrededor de 1600, difundido, posteriormente, por Europa gracias a la influencia de los músicos italianos. Se usó en la música cortesana en la que se ejecutaba la parte del bajo continuo, acompañando solistas o completando la parte armónica en los ensambles de cámara y pequeñas orquestas. En menor escala, a la tiorba se le dedicaron algunas páginas solistas de autores reconocidos como Giovanni Girolamo Kapsberger, Alessandro Piccinini o el célebre laudista alemán Sylvius Leopold Weiss (Remnant, 2002). De esta manera, durante los siglos XV y XVIII el laúd y sus variantes, entre estos la tiorba, fueron instrumentos de enorme popularidad en países como Inglaterra y Alemania. También participaban en la ejecución de la música sacra, solían integrar los ensambles y orquestas de la época junto al clavecín y las violas da gamba.

Las nuevas propuestas musicales, impulsadas, principalmente, por la Escuela de Mannheim y las novedosas posibilidades que, en materia de dinámica, ofrecía el recién inventado pianoforte sumieron en el olvido, al menos por casi tres siglos, al laúd y sus variantes. Por esto, en los períodos sucesivos, estos instrumentos renacentistas y barrocos, cayeron en desuso y sólo podían ser vistos en museos, colecciones o bodegas olvidadas de iglesias, palacios, monasterios, entre otros. Arnold Dolmetsch (1858-1940), probablemente, fue el primero en comenzar a desempolvar tanto la música antigua como sus instrumentos (Encyclopedia Britannica, s.f). Después de él, otros músicos e investigadores, en distintos países europeos, continuaron con esta labor que, en el presente, nos brinda un rico panorama de la historia musical en occidente, tanto en lo instrumental como en partituras y compositores.

Por una parte, el redescubrimiento de las obras monumentales de Bach y otros compositores anteriores al clásico, la decisiva participación de los lutieres -entre otros hechos- permitieron que músicos como Walter Gergwig, en la primera mitad del siglo XX, comenzaran a debelar el rico universo musical del laúd, los archilaúdes y la tiorba. Gracias a lo cual, estos instrumentos en la actualidad vuelven a contar con una cantidad considerable de ejecutantes, quienes, a su vez, han motivado a compositores a aventurarse a escribir nuevas obras. De forma similar, la guitarra estuvo fuera de las salas de concierto durante el Clasicismo y el Romanticismo por considerarla un instrumento técnicamente incapaz de ejecutar música compleja. En el Renacimiento, aun cuando tuvo mayor presencia en el ambiente de la música popular, algunos de los grandes vihuelistas le dedicaron hermosas composiciones. En el Barroco, en manos de Robert de Visée y Francesco Corbetta, deleitó la corte de Luis XIV de Francia. Posteriormente, Corbetta la llevó a Londres donde tuvo mucho éxito, mientras que, en esa misma época, el guitarrista español Gaspar Sanz, era reconocido como uno de los principales músicos de la época en ese país.

ESCENA. Revista de las artes, 2018, Vol. 78, Núm. 1 (julio-diciembre), pp. 161-178. ISSN 2215-4906 
La guitarra es un instrumento muy versátil, de bajo costo y fácil de trasladar, por esto, desde el Renacimiento ha sido el instrumento predilecto de la música popular en Occidente, esta particularidad, sin duda, facilitó su amplia distribución global. De esta manera, no es de extrañar su aparición en el lejano Japón. Según Coldwell, "the Japanese said to have been the first to own a guitar was Hiroshi Hiraoka (1856-1934) [Se dice que el primer japonés en tener una guitarra fue Hiroshi Hiraoka]" (Coldwell, 1997, p. 1). Como en el resto de países no europeos, la guitarra en Japón tuvo un desarrollo importante a partir de la segunda mitad del siglo XX, tanto en número y calidad de intérpretes como en la conformación de un nuevo tipo de ensamble instrumental: la "orquesta de guitarras".

Hiroki Niibori, en 1958, crea la primera orquesta de guitarras en el mundo. Su gusto por los grupos instrumentales y la guitarra, lo llevaron a crear nuevos tipos de guitarras con el objetivo de combinarlas y ampliar su registro y timbres. La orquesta de guitarras creada por el músico japonés, se compone de guitarras clavicémbalo, guitarras pícolo, soprano, alto, baja y guitarrón; combinadas pueden ejecutar obras del repertorio orquestal y sinfónico así como obras compuestas especialmente para este ensamble (Niibori Guitar World, s.f). Durante su visita a Japón, en 1980, el guitarrista y compositor argentino, Jorge Cardoso observa y escucha la Niibori Guitar Orchestra. Motivado por su riqueza tímbrica y ambiente, funda ese mismo año en Madrid la "Orquesta Iberoamericana de Guitarras". Años más tarde, uno de los integrantes de aquella orquesta el profesor, Luis Zumbado funda en la Escuela de Artes Musicales de la Universidad de Costa Rica, la "Orquesta de Guitarras de la Universidad de Costa Rica", grupo que ofrece su primera presentación el 17 de noviembre de 1982 (Zumbado, 2012).

El efecto mariposa producido por la Niibori Guitar Orchestra es evidente en el desarrollo de orquestas de guitarra alrededor del mundo. Además de la Orquesta Iberoamericana de Guitarras en España, como se mencionó, está la Orquesta de Guitarras de la Universidad de Costa Rica, la cual, a su vez, motivó la creación de la Tidewater Guitar Orchestra en Virginia, Estados Unidos; la Orquesta de Guitarras de la Fundación "María Escalón de Núñez", San Salvador, El Salvador; la Orquesta de Guitarras de la Etapa Básica de Palmares, Universidad de Costa Rica; la Orquesta de Guitarras del Conservatorio Castella, Heredia, Costa Rica; la Orquesta de Guitarras del Instituto Tecnológico de Costa Rica, Cartago, Costa Rica; la Orquesta de Guitarras de la Etapa Básica de Turrialba, Universidad de Costa Rica; la Orquesta de Guitarras de la Escuela Superior de Guitarra, San José, Costa Rica; la Orquesta de Guitarras de Costa Rica y la Orquesta de Guitarras de la Universidad Nacional, Heredia, Costa Rica (Zumbado, 2012). Además -y solo para citar algunos ejemplos- se cuenta con ensambles de este tipo en Alemania, México, Venezuela y Cuba, como "Las Sonantas Habaneras" de La Habana, Cuba y la Jugendguitarrenorchester de Baden, Württemberg, Alemania. Actualmente, el olvido de la guitarra ha sido subsanado y el instrumento goza de 
buena popularidad y saludable reputación en la música de concierto, también las orquestas de guitarra son apreciadas cada vez más por el gran público.

El tercer movimiento del concierto se basa en un tamborito chiricano. El tamborito chiricano fue la música más interpretada y asimilada por las y los habitantes de Puntarenas, desde la conformación de su primer asentamiento en el año de 1765 hasta 1930. A diferencia de la conservación de las tradiciones en otras provincias, este ritmo fue totalmente olvidado por el colectivo social puntarenense. Este hecho, por lo inusual, llama poderosamente la atención, máxime que los habitantes de esta provincia y, en especial, los de la cabecera de provincia, todavía resienten su desaparición.

Puntarenas, en sus inicios, fue poblada por habitantes provenientes de la provincia de Chiriquí, Panamá. Amador (2008) explica que la migración de las y los chiricanos se dio en tres etapas. Justamente, la tercera fue la que las y los llevó a Punta Burica, cabo Drake y todo el litoral Pacífico, lo que permitió alcanzar hasta el accidente geográfico entonces conocido como la "punta de arenas". El proceso colonizador de los chiricanos ocurrió en la segunda mitad del siglo XIX y terminó con la delimitación de la frontera en 1941. Este mismo autor sostiene que las razones de la migración chiricana obedecieron a la necesidad de ofrecer mejores pastos a su ganado, la destrucción de los pueblos indígenas en Chiriquí, el despojo de tierras, agotamiento de los suelos, incremento de la población y hasta los conflictos bélicos que conducían al alistamiento forzoso.

Los chiricanos trajeron a suelo costarricense, además de sus costumbres y tradiciones, el ritmo del tamborito chiricano, el cual es una mezcla entre lo africano y lo español. Los rasgos africanos se manifiestan en los instrumentos de percusión que participan en la ejecución y en el estilo antifonal del canto. La influencia española se nota en la forma en que acompañan las palmas al estilo de la música andaluza, particularmente, la sevillana. En el tamborito, el canto es responsabilidad de las mujeres. La voz principal, llamada "cantalante" propone el tema de la canción y, posteriormente, un coro de tres o más mujeres, cantan el estribillo. Todas marcan el ritmo y se acompañan con las palmas. Los hombres solo participan en la ejecución de los tres instrumentos de percusión usados en esta música: repicador, pujador y caja, este último recuerda la antigua caja, ahora redoblante, de los ensambles europeos (Zárate, 1985)².

Por más de 150 años, el tamborito alegró e hizo vibrar a las y los puntarenense. No obstante, la popularidad del tamborito chiricano puntarenense, el ejecutado con tambores, guitarra, marimba y quijongo, desaparece. Para el investigador García (2013), tres razones

${ }^{2}$ Para más detalles sobre el tamborito chiricano puntarenense véase Solera (2014).

ESCENA. Revista de las artes, 2018, Vol. 78, Núm. 1 (julio-diciembre), pp. 161-178. ISSN 2215-4906 
fueron la causa de su desaparición: en primer lugar, decretos que prohibieron su ejecución. En segundo lugar, la censura a manos de una élite social que buscaba la aprobación del Gobierno Central, por la manera provocativa y desvergonzada del baile. En tercer lugar, el arribo de nuevos ritmos a la ciudad de Puntarenas cuando en esta, se comenzaron a proyectar películas de producción extranjera o se intensifica el tránsito de músicos cubanos, colombianos y mexicanos por este puerto. De acuerdo con García, es comprensible que el centro y, específicamente, el Gobierno, censurara una manifestación vernácula. Por otra parte, y, posiblemente, con el objetivo de ser aceptados, a partir del arribo del cine y la visita de músicos de otros países, quizás las y los porteños decidieron abrazar estos nuevos ritmos y comenzaron a olvidar su tradicional y amado tamborito.

Además, otra posible razón para olvidar el tamborito debe agregarse a las expuestas por García, la del marcado sesgo misógino de la Costa Rica de finales del XIX y principios del XX, el cual todavía corroe la sociedad costarricense. Desde esta perspectiva, una manifestación musical, en la que la mujer, además de cantar, lidera la ejecución no podía ser aceptada en aquella sociedad misógina. Explica la estudiosa del folclore panameño, Dora de Zárate, sobre el papel de la mujer en el tamborito:

El canto siempre se ejecuta entre mujeres. Una con hermosa voz, cruda, natural, que es la solista o "cantalante" como la llamamos, ocupa su puesto al lado del cajero. Ella entona la melodía y le marca el compás, cantando el estribillo correspondiente al texto que va a cantar. El cajero la sigue y luego entran los otros tambores y simultáneamente, el coro de mujeres que dan palmadas apoyando el compás. Se establece una especie de diálogo entre la solista y su coro, actividad que continúa hasta que la "cantalante" deje la tonada, ya sea porque quiere cambiarla por otra o porque desea descansar o ser sustituida (Zárate, 1985, p. 1).

Las mujeres participan en el canto y entre ellas, la "cantalante" lo inicia, marca el tempo y a ella, tanto el ejecutante de la caja, como el resto de percusionistas, deben prestar toda la atención. Atención y liderazgo femenino que motivó, en la Puntarenas de la primera mitad del siglo XX, la censura y consecuente olvido de esta expresión musical. Con el propósito de mostrar el sesgo misógino de la sociedad costarricense, a continuación, se presentan dos casos de mujeres víctimas de dicho sesgo específicamente a principios del siglo XX.

Yolanda Oreamuno (1916-1956), "la primera escritora que expone y se rebela contra la situación de la mujer en la sociedad de nuestro país, en la primera mitad del siglo XX" (Editorial Costa Rica, 2016, p. 1), escribió:

La situación social de la mujer en Costa Rica viene a ser la raíz madre de lo que el Colegio [de Señoritas] llama con tanto acierto frivolidad ambiente. ... Desde que comienza la educación de nuestra mujer en el hogar se plantea ya su contradictoria situación: ¿Se educa

ESCENA. Revista de las artes, 2018, Vol. 78, Núm. 1 (julio-diciembre), pp. 161-178. ISSN 2215-4906 
a nuestras muchachas para que sean buenas señoras de casa, correctas esposas y fuertes madres, o se las educa para que tomen una activa parte en el conjunto social, dentro y fuera del hogar? (Oreamuno, 1936).

Sobre el problema del rol de la mujer y, específicamente, sobre la paradoja de la educación de la mujer en Costa Rica, descrita, anteriormente, por la escritora, la psicología del siglo XX ha investigado ampliamente, obteniendo como resultado que, este rol ha sido inducido por la misma sociedad, la religión (principalmente la Iglesia Católica en Costa Rica) y la cultura. Así, busca inventar la idea de concebir a la mujer como la madre idílica, quien de acuerdo con Jodorowsky y Costa (2011), debe ser protectora del hogar, dadora de alimento, infatigable, en alerta constante y con obligación de ser dulce, elegante y digna. En la Costa Rica que le tocó vivir a Oreamuno, cuando una mujer hacía evidente esa paradoja y, al educarse, se sentía con los mismos derechos del hombre, la sociedad la despreciaba y, consecuentemente, tendía a aislarla, silenciarla y, posteriormente, olvidarla.

Oreamuno, casada en primeras nupcias con el Embajador de Chile en Costa Rica, logra salir de la asfixiante sociedad costarricense y traslada su residencia a Chile. Al fallecer su primer esposo, regresa a Costa Rica y aquí se casa por segunda vez. Luego del divorcio, se traslada a Guatemala donde vive por algunos años y termina sus días en México, en casa de otra exiliada costarricense, la poeta y escritora Eunice Odio, donde muere en 1956, a la corta edad de 40 años. Fue sepultada en la Ciudad de México. Cuatro años después, sus restos fueron traídos a Costa Rica para depositarlos en el Cementerio General de San José. Su tumba permaneció 50 años sin inscripción alguna, hasta que, en el año 2011, a los 55 años de haber fallecido, finalmente, le fue colocada una placa conmemorativa.

Las preguntas que emergen son: ¿por qué partió del suelo patrio esta escritora?, ¿por qué este aparente desprecio en el trato a sus restos? y ¿por qué sus cuarenta años de vida están claramente divididos en dos períodos: hasta los 20 años, el período de joven hermosa, talentosa y popular y después de los 20 años, el período de la tragedia, soledad y enfermedad? Hablan tiorba y guitarra en el concierto: una posible respuesta a tales preguntas, tanto para el caso de Oreamuno, como para otras grandes mujeres costarricenses como Eunice Odio, María Isabel Carvajal y Rocío Sanz, entre otras, fue la de no encajar en la sociedad ultraconservadora de la Costa Rica de la primera mitad del siglo XX. Yolanda Oreamuno, al atreverse a preguntar en su juventud, para qué se educan las mujeres en Costa Rica, la sumió en el inmerecido exilio impuesto por la misoginia social costarricense de la primera mitad del siglo XX.

Así también, no encajó Isabel "Chavela" Vargas Lizano en aquella sociedad. A ella, el Concierto de Chira desea rescatarla del olvido en el tercer movimiento. Decidió autoexiliarse en México y desde ahí, además de desarrollar su arte, guardó, durante toda su vida, un gran

ESCENA. Revista de las artes, 2018, Vol. 78, Núm. 1 (julio-diciembre), pp. 161-178. ISSN 2215-4906 
resentimiento contra su país natal. El reclamo del tamborito porteño y el rencor de Chavela cantan en el tercer movimiento. A Chavela, se le hizo imposible desarrollar su talento y mucho menos manifestar su lesbianismo en la Costa Rica que la vio nacer. Fue una mujer contestataria e independiente que, tan solo quería ser libre y, precisamente, su búsqueda de libertad la hizo partir de este país, al cual, con razón, alguna vez describió como: "la negación del mundo. ... Qué país Costa Rica. Yo pondría allí a todos los suicidas del mundo. Les pondría allí un departamento. Sería un buen negocio una tienda de ataúdes. Eso es lo que pienso de Costa Rica" (Ordaz, 2009, p. 1).

La sociedad costarricense de buena parte del siglo XX y épocas anteriores, seguía el orden impuesto por la Iglesia Católica, en el cual la realización femenina se consumaba en el hogar; en la atención a las y los hijos; en la atención al hombre, ya sea padre o esposo y en ser madre. La mujer crítica, pensante y, sobre todo, libre, era estigmatizada. Por eso el rencor de Chavela, la partida de Yolanda y el sufrimiento de tantas mujeres brillantes en este país.

Con justicia, se debe reconocer los esfuerzos de algunas instituciones, especialmente, a partir de la última década del siglo pasado, por revertir este orden y por tratar de llegar a una igualdad real de género en el presente. No obstante, y con vergüenza, todavía se lee en el artículo 75 de la Constitución Política de la República de Costa Rica: "la Religión Católica, Apostólica y Romana, es la del Estado, el cual contribuye a su mantenimiento, sin impedir el libre ejercicio en la República de otros cultos que no se opongan a la moral universal ni a las buenas costumbres" (Constitución Política de la República de Costa Rica, 1949, p. 10). Poco o nada puede avanzar una nación en el tema de la igualdad real de género, si el Estado se declara confesor de una religión que relega y anula el papel de la mujer en la construcción de la realidad y de la sociedad.

\section{El concierto}

El concierto está estructurado en tres movimientos para ser ejecutados sin interrupciones, cuyo propósito es el de no interrumpir el discurso con pausas innecesarias. El discurso musical muestra características propias del barroco, período en la que la tiorba tuvo su apogeo, y el jazz. El primer movimiento es una elaboración libre sobre el "bajo caminante" (walking bass), estilo de ejecución del contrabajo en las obras de jazz. El segundo movimiento, también recurre a otro elemento distintivo del jazz, la improvisación; el uso del saltillo (combinación rítmica de corchea con punto-semicorchea) y la improvisación, son recursos ampliamente usados en la música barroca. Ambas características se introducen en la partitura con el propósito de evidenciar esta relación indisoluble entre la tiorba y ese período musical. La improvisación, el sentimiento de libertad que tiene el o la instrumentista 
en la interpretación, la illégalité ejecución rítmica particular de figuras de igual valor en el barroco, similar al actual swing del jazz y la ornamentación, son características que unen a estos estilos sin importar los siglos que los separan. La Figura 1 muestra el material temático que unifica la obra: cuatro negras que conducen a ocho corcheas en movimiento de segunda. La Figura 2, el contrapunto sobre un bajo caminante como tema del primer movimiento.

Figura 1. Material temático

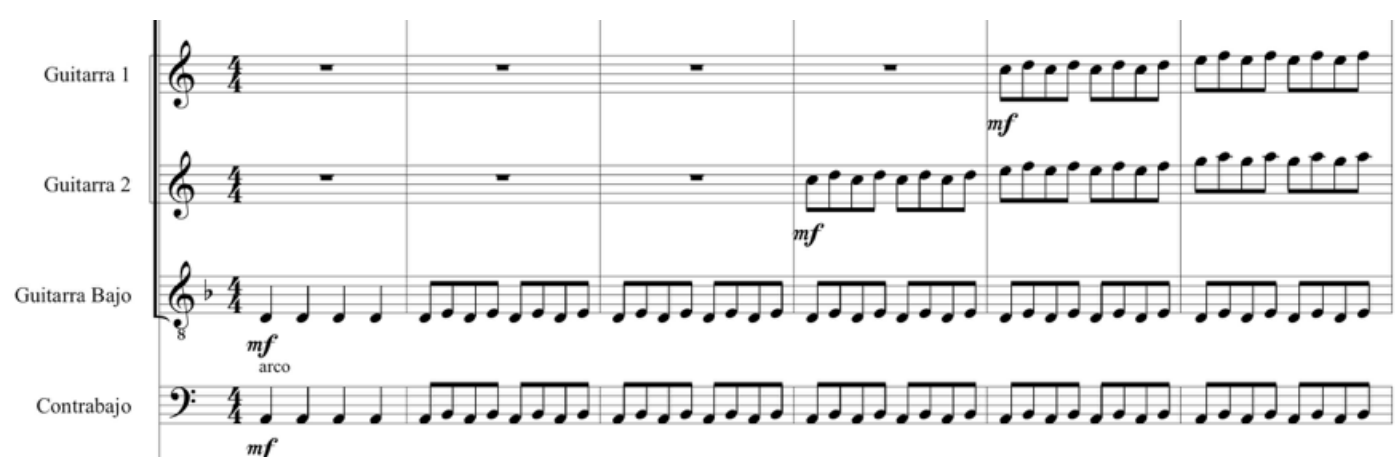

Fuente: partitura del Concierto de Chira.

Figura 2. Tema del primer movimiento

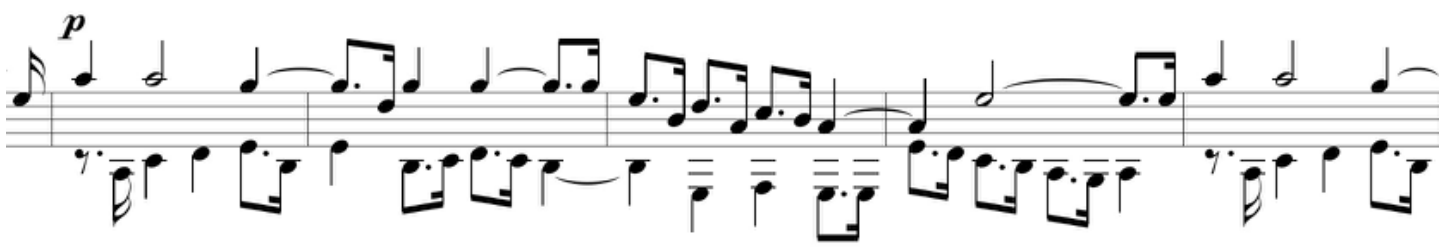

Fuente: partitura del Concierto de Chira.

El segundo movimiento, en su forma original, fue compuesto en el 2001, en versión para guitarra y piano. Fue estrenado en el Día Internacional de la Mujer celebrado ese mismo año. Recuerda un bolero, con las libertades propias en la ejecución instrumental de una danza hecha para escuchar y no para bailar. La Figura 3 muestra el tema del bolero, en el compás 213, en la parte de la tiorba. Entre el segundo movimiento y el tercero la tiorba en solitario ejecuta el villancico renacentista "De los álamos vengo, madre" de Juan Vásquez, cuyo propósito es recordar que las culturas latinoamericanas existen a partir de procesos continuos de hibridación. La Figura 4 muestra el inicio de la exposición de la célebre canción renacentista en el compás 307. 
Figura 3. Bolero, tema del segundo movimiento

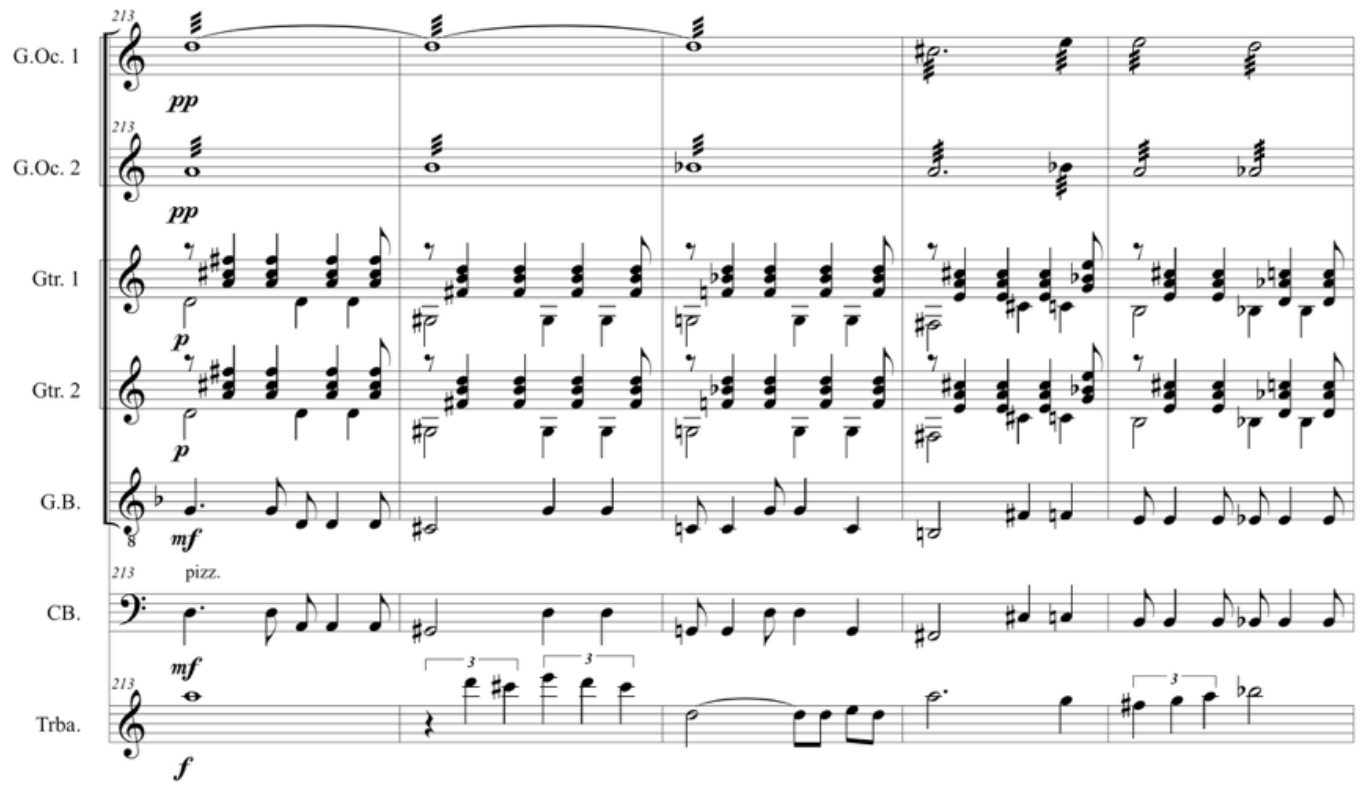

Fuente: partitura del Concierto de Chira.

Figura 4. Exposición del villancico "De los álamos vengo, madre"
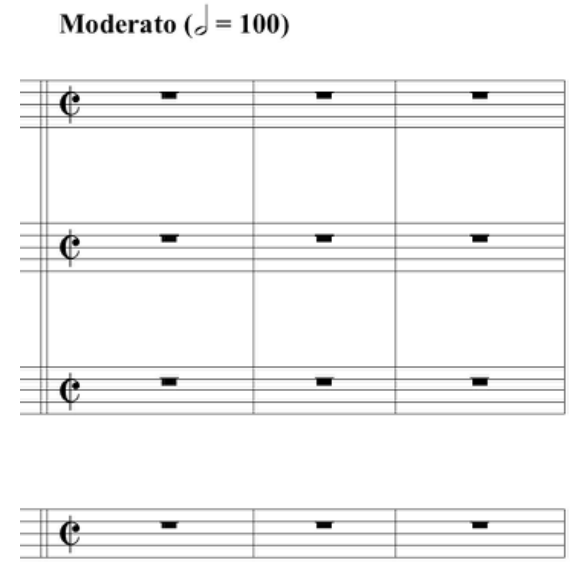

...pues que "de los álamos vengo"

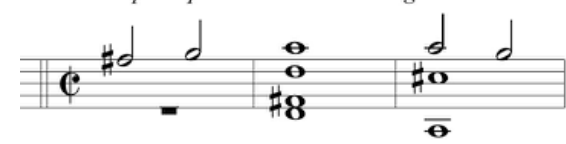

Fuente: partitura del Concierto de Chira. 
Comienza el tercer movimiento con la indicación de "Tamborito porteño". Es preciso indicar que, "porteña, porteño" es el topónimo de las y los habitantes de la ciudad de Puntarenas. Las y los habitantes del resto de puertos, inclusive los de Limón, principal puerto en el Caribe, no usan este topónimo. De aquí la indicación del movimiento haciendo referencia al extinto tamborito chiricano puntarenense. El rasgueo de la guitarra es una elaboración propia que supone cómo pudo haberse interpretado este ritmo con guitarra en Puntarenas, por cuanto el tamborito chiricano original no usa este instrumento. La Figura 5 muestra el inicio del tercer movimiento, en el compás 454 y el rasgueo del compás 462. En el compás 475 se recuerda a Chavela Vargas por medio de la inserción del tamborito chiricano "Se va la Chavelita", como lo muestra la Figura 6.

Figura 5. Inicio del tercer movimiento
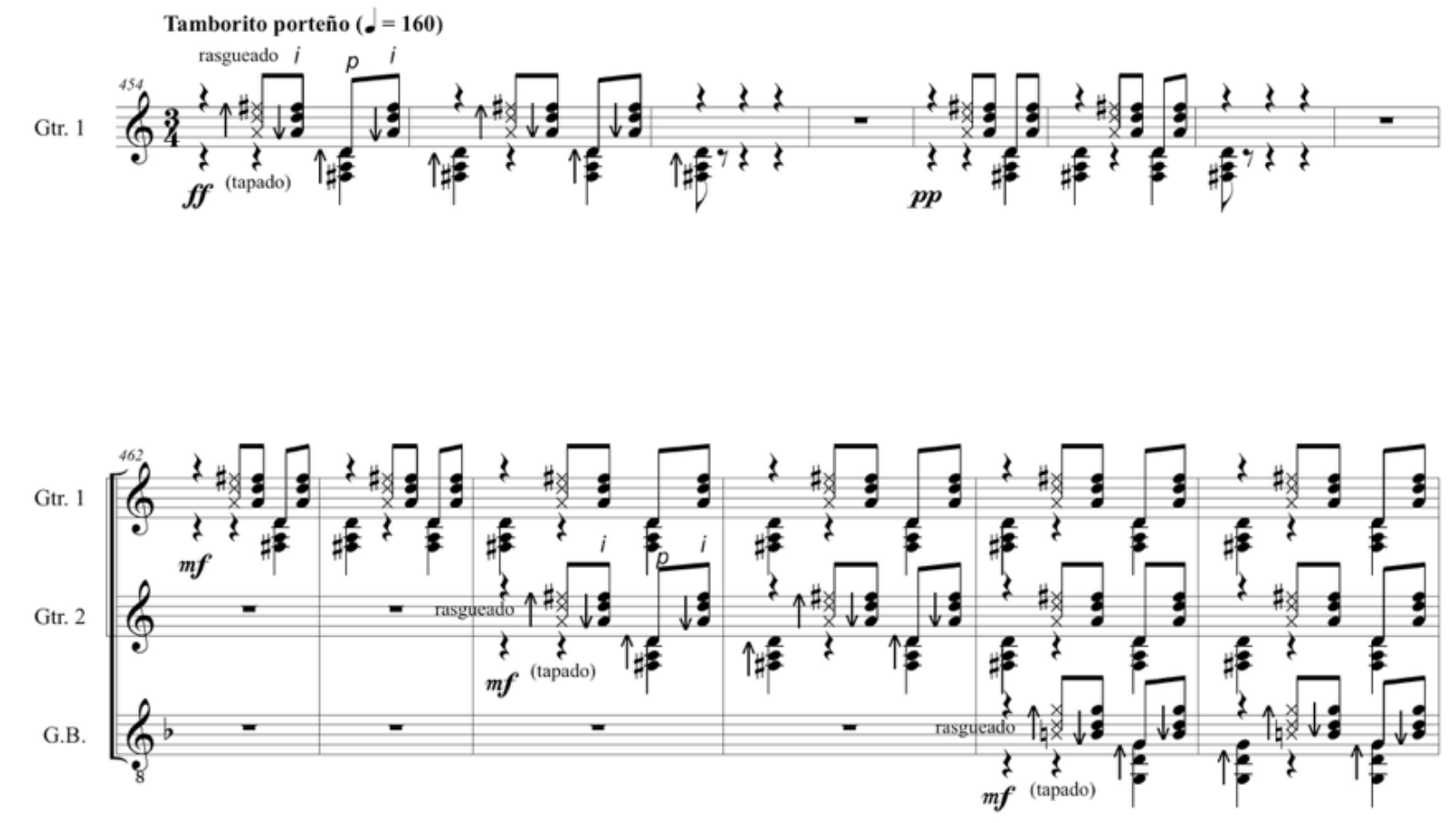

Fuente: partitura del Concierto de Chira. 
Figura 6. Tamborito chiricano "Se va la Chavelita", en la tiorba

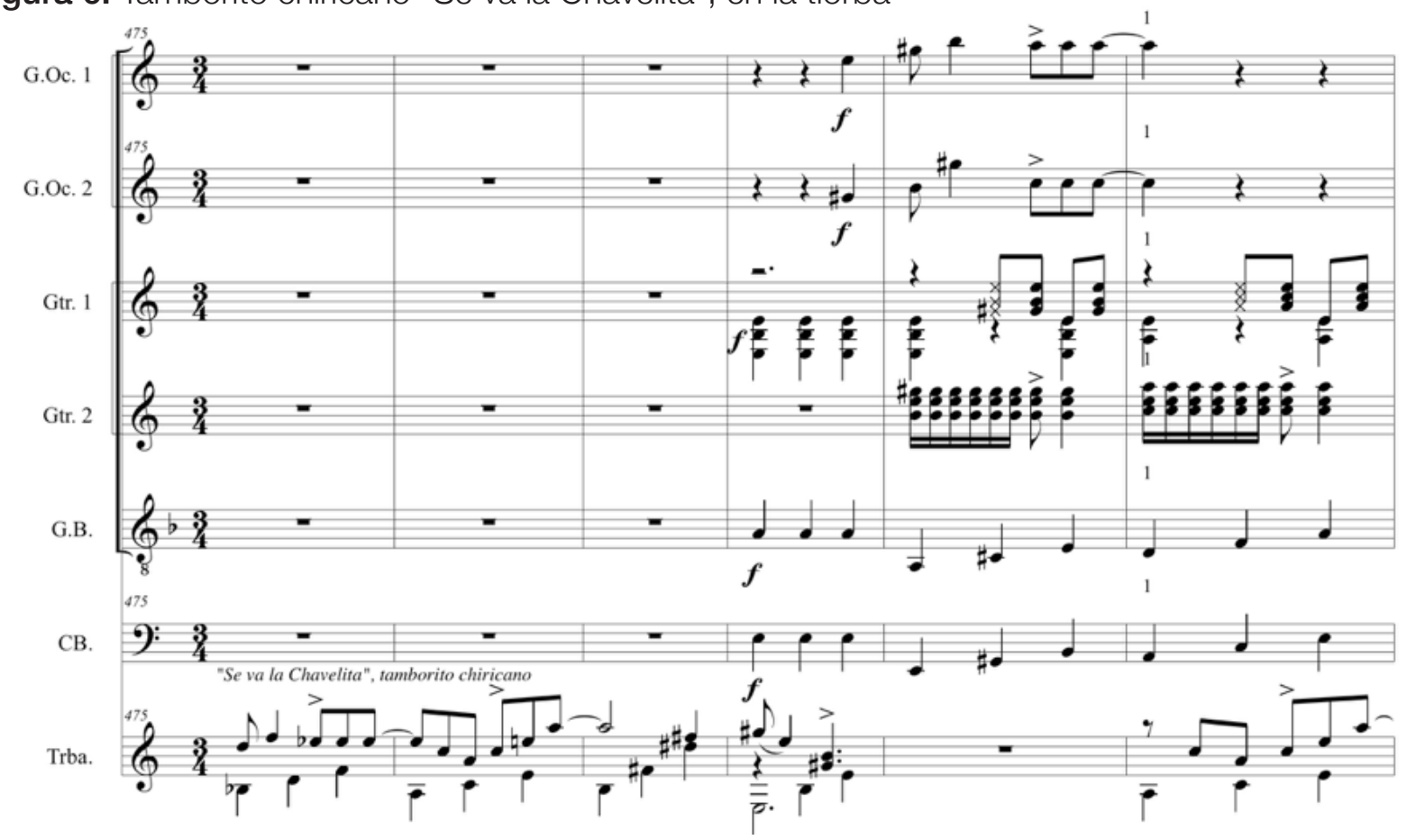

Fuente: partitura del Concierto de Chira.

\section{Conclusión}

El análisis no formal del concierto, sino arqueológico, desde una perspectiva foucaultiana, da cuenta de un hilo conductor subyacente: el del olvido, ya sea por temor al cambio o por menospreciar, estigmatizar y censurar. Esto se evidencia desde la selección de los instrumentos con los cuales se interpreta la obra: la tiorba y la guitarra; instrumentos olvidados y desconocidos por muchos años en el ambiente de la música de concierto. La intertextualidad de la obra ofrece un espacio para reflexionar sobre los territorios patrios olvidados y el olvido de tantas insignes mujeres costarricenses.

La historia de las provincias periféricas costarricenses tiene como común denominador las imposiciones dictadas por el centro de poder, ubicado en el Valle Central. De esta manera, la historia de Costa Rica se desenvuelve en una relación centro-periferia. El centro, desde la independencia, y hasta hace poco, viene ejerciendo el poder para definir cómo deben ser las y los costarricenses, con lo cual ha dejado por fuera de ese "ser costarricense" una enorme cantidad de rasgos, costumbres y culturas propias de este país. Lejos ha estado el Valle Central de ver al país como, expone Anderson (1993), "una comunidad políticamente imaginada como inherentemente limitada y soberana” (p. 23). 
Finalmente, el olvido es también silencio. El pasado se ha silenciado y es tiempo de iniciar procesos de reversión en todas las aristas, pero, principalmente, en las de educación y la cultura, para enseñar nuestro pasado híbrido y abrazar las epistemologías del sur y los feminismos decoloniales. Esto con el fin de erradicar, por siempre, la amnesia crónica y actitud silenciadora de la sociedad costarricense.

\section{Referencias}

Amador, J. (2008). Historia y tradición en Potrero Grande un pueblo costarricense de origen chiricano-panameño. San José: Editorial de la Universidad Estatal a Distancia.

Anderson, B. (1993). Comunidades imaginadas. Reflexiones sobre el origen y la difusión del nacionalismo. México, D.F.: Fondo de Cultura Económica.

Encyclopedia Britannica. (s.f.). Arnold Dolmetsch. Recuperado de https://www.britannica. com/biography/Arnold-Dolmetsch

Coldwell, R. (1997). The Early Guitar in Japan. Recuperado de http://www.guitarandluteissues.com/early-j.htm

Constitución política de Costa Rica. (1949). Recuperado de http://pdba.georgetown.edu/ Parties/CostaRica/Leyes/constitucion.pdf

Editorial Costa Rica. (2016). Yolanda Oreamuno. Recuperado de http://www.editorialcostarica.com/escritores.cfm?detalle=1074

Ferrero, L. (2002). Mil y tantos tiquismos (Costarricensismos). San José: Editorial de la Universidad Estatal a Distancia.

Fumero, P. (2005). Cultura y sociedad en Costa Rica 1914-1950. San José: Editorial de la Universidad de Costa Rica.

García, P. (2013). Historia de las artes escénicas puntarenenses en el marco de la identidad cultural (tesis para optar al grado de Maestría Académica en Artes). Universidad de Costa Rica, San José.

Ibarra, E. (2001). Fronteras étnicas en la conquista de Nicaragua y Nicoya. Entre la solidaridad y el conflicto 800 d.C.-1544. Recuperado de https://goo.gl/PGu6Ze

Instituto Nacional de Estadística y Censo (INEC). (2012). X Censo Nacional de Población y VI de Vivienda 2011. Resultados Generales. San José: Instituto Nacional de Estadística y Censos. Recuperado de http://www.cipacdh.org/pdf/Resultados_Generales_Censo_2011.pdf

ESCENA. Revista de las artes, 2018, Vol. 78, Núm. 1 (julio-diciembre), pp. 161-178. ISSN 2215-4906 
Isla de Chira. (s.f.A). Información. Recuperado el 28 de agosto de 2016, a partir de http:// chiracr.com/Chiralslandlnformation.htm

Isla de Chira. (s.f.B). Información e historia. Recuperado de http://chiracr.com/marcopolitico.htm Isla de Chira. (s.f.C). Información e historia. Recuperado de http://chiracr.com/Informationiii.htm Isla de Chira. (s.f.D). Información e historia. Recuperado de http://chiracr.com/instituciones.htm Isla de Chira. (s.f.E). Información e historia. Recuperado de http://chiracr.com/marconarrativo.htm Jodorowsky, A. (2009). La danza de la realidad (Psicomagia y psicochamanismo). Madrid: Ediciones Siruela S.A.

Jodorowsky, A., y Costa, M. (2011). Metagenealogía. Madrid: Ediciones Siruela S.A.

Kaufman, T. (1991). The history of the Nawa language group from the earliest times to the sixteenth century: somo initial results. Recuperado de http://www.albany.edu/anthro/ maldp/Nawa.pdf

Niibori Guitar World. (s.f.). Dr. Hiroki Niibori. Recuperado de http://english.niibori.com/profile/ profile_e.html

Ordaz, P. (10 de mayo del 2009). Chavela Vargas. "Así me voy a morir, libre, sin yugos". El País Semanal. Recuperado de http://elpais.com/diario/2009/05/10/ eps/1241936812_850215.html

Oreamuno, Y. (1936). ¿Qué hora es? Medios que usted sugiere al Colegio para librar a la mujer costarricense de la frivolidad ambiente. Recuperado de https://goo.gl/PTpR2J

Remnant, M. (2002). Historia de los instrumentos musicales. Barcelona: Ma non troppo.

Solera, M. (2014). Tamborito chiricano puntarenense. Istmica, (17), 45-63.

Vitoria, F. (2004). Pedrarias Davila. El valiente. Recuperado a partir de http://webs.ono.com/ sancristobaldecuellar/historiaSC/PEDRARIAS.pdf

Zárate, D. (1985). El Tamborito. Diario la Prensa. Panamá.

Zumbado, L. (2012). Orquesta de guitarras de la Universidad de Costa Rica: a propósito del 30 Aniversario de su fundación. Revista Káñina, 36(1), 255-274. 